\title{
Avaliação dos parâmetros morfológicos da qualidade de mudas de Paubrasilia echinata (pau-brasil) em viveiro florestal
}

Evaluation of morphological parameters for seedling quality of Paubrasilia echinata (paubrasil) in forest nursery

\author{
S. H. M. Gomes ${ }^{1 *}$; F. B. Gonçalves²; R. A. Ferreira ${ }^{3}$; F. R. M. Pereira ${ }^{4}$; M. M. J. \\ Ribeiro $^{3}$ \\ ${ }^{1}$ Centro de Métodos Quantitativos, Universidade de São Paulo, 13418-900, Piracicaba-São Paulo, Brasil \\ ${ }^{2}$ Laboratório de Ecologia Florestal, Universidade Federal de Sergipe, 49100-000, São Cristóvão-Sergipe, Brasil \\ ${ }^{3}$ Departamento de Ciências Florestais, Universidade Federal de Sergipe, 49100-000, São Cristóvão-Sergipe, Brasil \\ ${ }^{4}$ Departamento de Ciências Florestais, Universidade Federal da Paraíba, 58051-900, João Pessoa-Paraíba, Brasil
}

*silviohgomes@yahoo.com.br

(Recebido em 04 de julho de 2017; aceito em 18 de dezembro de 2018)

\begin{abstract}
Paubrasilia echinata é uma espécie arbórea, perenifólia, de ocorrência em florestas da mata atlântica e possui madeira densa e resistente, ameaçada de extinção devido a sua exploração inadequada. Tendo em vista a importância econômica e ambiental, se faz necessário a produção de mudas com parâmetros de qualidade aceitáveis, com crescimento análogo e, consequentemente, menor mortalidade no campo. Assim, o presente trabalho teve como objetivo realizar análise morfológica para qualidade de mudas de pau-brasil, além do ajuste de equações para estimativa do Índice de qualidade de Dickson (IQD), Peso da matéria seca da parte aérea (PMSPA) e Peso da matéria seca da raiz (PMSR) como alternativa ao uso de métodos destrutivos para a espécie. Foram realizadas três avaliações quinzenalmente com 50 amostras das 540 mudas de pau-brasil. As variáveis utilizadas foram: diâmetro a altura do colo (DAC), altura $(\mathrm{H})$ e o número de folhas (NF), peso seco e úmido de 10 mudas em cada avaliação. Foram testadas 15 equações, visando gerar o modelo geral para todas as variáveis de interesse. Para o DAC e $\mathrm{H}$, as mudas obtiveram valores acima de $5 \mathrm{~mm}$ e $20 \mathrm{~cm}$, respectivamente. Observou-se que o peso de massa seca, na relação PMSPA/PMSR o valor médio alcançado foi de 3,24 e o IQD de 0,53. As melhores equações com ajustes para IQD foram de 3 a 6. As melhores equações para PMSPA e PMSR, respectivamente, foram a 12 e 15 . Conforme os parâmetros morfológicos apresentados, as mudas de pau-brasil estão aptas ao plantio mesmo apresentando baixo IQD.
\end{abstract}

Palavras-chave: Heliófitas, Índice de Qualidade de Dickson, Modelos lineares.

Paubrasilia echinata is a perennial species occurring in forests of Atlantic forest and has a dense and resistant wood, threatened with extinction due to its inadequate exploitation. Considering the economic and environmental importance, it is necessary to produce quality seedlings with good parameters, which show a uniform growth and a lower percentage of mortality in the field. Thus, the present work had as objective to perform a morphological analysis for quality of pau-brasil seedlings produced in the nursery of the Federal University of Sergipe, besides the adjustment of equations for estimation of Dickson's Quality Index (DQI), dry shoot weight (DSW) and dry root weight (DRW) as an alternative to the use of destructive methods for the specie. Three evaluations were carried out biweekly with 50 samples of the 540 sapwood seedlings produced. The variables used were: diameter at neck height $(\mathrm{DNH})$, height $(\mathrm{H})$ and number of leaves (NL), dry and wet weight of 10 seedlings at each evaluation. We also tested 15 equations, aiming to generate the general model for all variables of interest. For the DAC and $\mathrm{H}$, the seedlings obtained values above $5 \mathrm{~mm}$ and $20 \mathrm{~cm}$ respectively. It was observed that the dry mass weight, in the DSW/DRW ratio, the mean value reached was 3.24 and the DQI was 0.53 . The best equations for PMSPA and PMSR, respectively, were at 12 and 15 . According to the morphological parameters presented, the pau-brasil seedlings are suitable for planting even though it presents a low DQI.

Keywords: Heliophites, Dickson Quality Index, linear models. 


\section{INTRODUÇÃO}

O pau-brasil, pertencente à família Fabaceae (Leguminosae) e subfamília Caesalpinioideae, é espécie nativa do Brasil, é propagada naturalmente na floresta pluvial atlântica nos estados compreendidos desde o Rio Grande do Norte até o Rio de Janeiro [1, 2]. Apresenta seiva de cor vermelha, muito utilizada para tingir tecidos e algodão ao longo da história de colonização do país. Atualmente sua madeira é destinada principalmente a fabricação de arcos de violino de alta qualidade [3].

Foi descrita em 1785 como Caesalpinia echinata por Lamarck, porém estudos filogenéticos recentes realizados por Gagnon et al. (2016)[3], relativos a comparação de sequências de DNA do pau-brasil com 200 outras plantas geneticamente próximas, também provenientes dos trópicos, revelou que a árvore tipicamente brasileira representa linhagem evolutiva distinta e única, o que lhe confere o direito de possuir um gênero próprio caracterizado Paubrasilia, passando então a ser chamada e descrita Paubrasilia echinata (Lam.) E. Gagnon, H. C. Lima \& G. P. Lewis. Segundo os mesmos autores, Paubrasilia foi derivado da "latinização" do seu nome popular, em virtude da importância da espécie para o Brasil.

O pau-brasil vem sendo explorado de forma exacerbada desde o período da colonização do Brasil, logo, a transformação do bioma Mata Atlântica é resultado desse processo histórico. A perda de sua biodiversidade prosseguiu durante os períodos que marcaram a introdução da cana de açúcar no país, ciclo do ouro, plantação de cafezais, produção de carvão vegetal, extração de madeira, e implantação de pastagens, produção de papel e celulose, com a instalação de assentamentos de colonos, construção de barragens e rodovias, além da urbanização desordenadamente intensa [4].

Apesar de toda a sua importância ecológica, histórica e sociocultural, o pau-brasil encontra-se na Lista Vermelha da Flora Brasileira [5]. Estudos sobre espécies ameaçadas de extinção são prioritários para definir estratégias de conservação [6], o que justifica aprimorar técnicas para produção de mudas que possuam crescimento potencial à sua permanência vital no campo e, consequentemente, menor inevitabilidade de reposição.

O pau-brasil, além de sofrer fortes pressões antrópicas é uma espécie que apresenta características intrínsecas que têm sido um entrave à sua regeneração, como a baixa viabilidade natural das suas sementes [7]. Os níveis de glicose e frutose, em relação aos níveis de sacarose em período de tempo rápido, têm sido apontados como as principais causas para a perda rápida do poder de germinação das sementes desta espécie [8]. Soma-se a isso, a dificuldade no enraizamento de seus propágulos quando se utiliza o método de propagação por estacas, limitando assim seu uso, exigindo deste modo, estudos mais específicos e detalhados de rejuvenescimento, condições ambientais, substratos e outros aspectos ligados a propagação vegetativa $[9,10]$.

Nos últimos anos tornou-se crescente a produção de mudas de espécies florestais naturais, em virtude da necessidade de suprir a demanda de reflorestamentos e da recomposição de áreas degradadas, visando diminuir os impactos ambientais causados pelos intensos desmatamentos [11]. No cultivo de espécies nativas, a produção de mudas é de extrema importância, sobretudo na preocupação em avaliar o tipo de substrato como foco para o seu desenvolvimento [12, 23]. Especialmente atrelado ao nível de luminosidade, que também é considerado um dos fatores de grande relevância a serem considerados para o crescimento das mudas [16, 22]. Esta última tem sido destacada como fator limitante ao seu pleno desenvolvimento, já que as espécies deste tipo possuem características intrínsecas para seu crescimento [12].

De acordo com Caldeira et al. (2008) [13], a produção de mudas de espécies florestais em larga escala para diversos tipos de plantios comerciais, recuperação de áreas degradadas e recomposição de florestas ou de fragmentos, favorece a procura por alternativas que diminuam os custos de manejo dessas espécies. Logo, mudas que possuem bons parâmetros de qualidade são importantes para o sucesso no estabelecimento e desenvolvimento dos povoamentos com espécies nativas [23].

Segundo Gomes (2001) [14], há grande necessidade de se produzir mudas de espécies florestais em viveiros, levando em conta que deve existir maior proteção na fase inicial e de manejos específicos. Deste modo, é possível ter maior padronização no crescimento tanto da altura, quanto do sistema radicular, promovendo assim maior rustificação, que, após o plantio no 
campo, permita-se obter maior vigor às condições impeditivas do meio e as mudas crescerem satisfatoriamente.

No processo de prescrição da qualidade das mudas para serem utilizadas nos replantios, podem ser empregadas características tanto morfológicas, que são baseadas nos aspectos fenotípicos, quanto fisiológicas. As primeiras ainda são as características mais recorrentemente utilizadas para atribuir qualidade às mudas, por ter maior aceitação dos produtores em viveiro, necessitando assim de mais definições que possam responder à sobrevivência e ao crescimento inicial, em função das adversidades que são encontradas em campo após o plantio [15]. Nesse sentido, experiências concluem que essas características adquiridas em viveiro são importantes para o sucesso do desempenho das mudas em situações de campo [17].

De acordo com Chaves e Paiva (2004) [16] os parâmetros morfológicos, em virtude de sua compreensão intuitiva por parte dos viveiristas, são mais utilizados para determinação do padrão de qualidade das mudas, sobretudo quando comparados aos fisiológicos que são de difícil mensuração e análise.

Os parâmetros morfológicos mais utilizados para caracterizar qualidade de mudas florestais são a altura, diâmetro de colo, relação altura sobre diâmetro, massa aérea, radicular e total. Esses parâmetros são determinados por fatores genéticos e ambientais que promovem o crescimento das plantas [18]. Entretanto, alguns desses parâmetros exigem a destruição da muda produzida, a exemplo da determinação da massa aérea e radicular, para cálculo do IQD (Índice de Qualidade de Dickson), que é um importante indicador de robustez da muda [19].

Uma alternativa ao uso de métodos destrutivos para determinação de algumas variáveis de difícil aquisição em estudos de qualidade de mudas é o ajuste de modelos lineares através de regressão. Esses modelos tentam explicar a variável a ser estimada através de outras variáveis de fácil aquisição, a exemplo do diâmetro e altura e, se há ou não relação entre elas. Estas equações são eventualmente utilizadas para estimativas de algumas variáveis em árvores em pé, para quantificação da altura, volume e biomassa [20, 21]. No entanto, alguns trabalhos tem se empenhado em estimar mais de uma variável dependente em apenas uma equação. Isto é possível graças a utilização de variáveis binárias (dummy), conhecidas também como variáveis fictícias ou binárias.

Com o pau-brasil, Aguiar et al. (2011) [22], testaram diferentes níveis de sombreamento, no qual foi constatado que a espécie possui atributos de plantas heliófilas em que, nas condições de maior luminosidade, as mudas obtiveram maior crescimento e melhor relação do sistema radicular e aéreo, sobretudo para o IQD.

O trabalho teve como objetivo avaliar a qualidade de mudas de pau-brasil Paubrasilia echinata (Lam.) produzidas em viveiro florestal através de parâmetros morfológicos (altura, diâmetro a altura do colo e número de folhas). Além do ajuste de equações matemáticas utilizando a variável binária, para predição do Índice de Qualidade de Dickson (IQD), peso da massa seca da parte aérea (PMSPA), peso da massa seca da raiz (PMSR) e peso da massa seca total (PMST).

\section{MATERIAL E MÉTODOS}

As mudas foram produzidas no viveiro florestal do Campus da Universidade Federal de Sergipe no município de São Cristóvão - SE, situada nas coordenadas $10^{\circ} 55^{\prime} 32^{\prime \prime}$ S e $37^{\circ} 06^{\prime} 08^{\prime \prime} \mathrm{W}$, sob o clima tropical chuvoso com verão seco e períodos chuvosos entre os meses de abril a agosto, tipo As de Köppen, atingindo temperatura média anual de $25,5^{\circ} \mathrm{C}$ e umidade relativa do ar média de $75 \%$ e precipitação média de $1.200 \mathrm{~mm}$ [29].

As sementes de Paubrasilia echinata (Lam.) foram semeadas inicialmente em sementeira, onde o substrato compõe frações de areia, composto de terra de subsolo (areia preta), areia (areia lavada) e esterco bovino na proporção 3:1:1. A emergência da plântula ocorreu de 3 a 4 dias após a semeadura.

A adubação foi realizada logo após a retirada do telado (sombrite 50\%), após 15 dias. A cada metro cúbico de substrato foram acrescidos $5 \mathrm{~kg}$ de superfosfato simples e $500 \mathrm{~g}$ de cloreto de potássio e $300 \mathrm{~g}$ de FTE. Após a primeira adubação as mudas receberam adubação em cobertura semanalmente de acordo com a característica do grupo ecológico da espécie manejada. Foram usados $60 \mathrm{~g}$ de cloreto de potássio e $25 \mathrm{~g}$ de sulfato de amônio diluídos em $10 \mathrm{~L}$ de água [31]. O 
recipiente utilizado foi saco de polietileno de 10 x $20 \mathrm{~cm}$ e a irrigação feita durante 3 vezes ao dia.

Foram realizadas três avaliações quinzenalmente a partir do lote contendo 540 mudas em pleno sol. A cada avaliação foram sorteadas aleatoriamente 50 mudas dentro do lote, obtendo-se dados de altura $-\mathrm{H}(\mathrm{cm})$ e diâmetro a altura do colo - DAC $(\mathrm{mm})$. Ao término das avaliações, foram selecionadas 10 mudas das 50 sorteadas para determinar a massa seca da parte aérea (PMSPA) e da raiz (PMSR).

Para determinação do peso seco, tanto da parte aérea quanto da raiz, foram separadas em sacos de papel e colocadas em estufa a $65^{\circ} \mathrm{C}$ por $48 \mathrm{~h}$, com circulação forçada de ar. Esta aferição foi realizada utilizando-se balança Bel Energineering (mark:2200; máx:2200 g; mín:200 mg; d=10 $\mathrm{mg}$; e: $100 \mathrm{mg}$; classe II; 220V). Para realização das medições no viveiro foram utilizadas régua e paquímetro para as alturas e diâmetros, respectivamente. A altura das mudas foi mensurada a partir da superfície do solo até a última gema apical.

O Índice de Qualidade de Dickson (IQD) é bastante empregado na avaliação de qualidade de mudas, por considerar o vigor e o equilíbrio da distribuição da biomassa na muda e por contemplar diversos parâmetros como o peso de massa seca da parte aérea (PMSPA), da raiz (PMSR), a relação altura/diâmetro e PMSPA/PMSR. Quanto maior for o valor do índice melhor será a qualidade da muda. Foi obtido pela expressão:

$$
I Q D=\frac{\text { PESO MATÉRIA SECA TOT AL }}{\frac{\text { Altura }(\mathrm{cm})}{\text { Diâmetro }(\mathrm{mm})}+\frac{\text { Peso Matéria Seca Parte Aérea }(\mathrm{g})}{\text { Peso Matéria Seca Raiz }(\mathrm{g})}}
$$

O ajuste dos modelos propostos foi dividido em duas etapas. Na primeira foram determinadas seis equações para estimativas do IQD, por ser a variável mais importante e, seleção da melhor equação. Na segunda etapa, foram ajustadas as mesmas equações com as variáveis binárias e, assim, gerar equações gerais capazes de predizer todas as variáveis de interesse (Tabela 1). As variáveis dummy possuem caráter prático interessante por se tratar de uma única equação com coeficientes que controlam alguma variável de interesse em uma única expressão, por meio da ativação ou não desses coeficientes (desativado quando 0 ou ativado quando 1 ). $\mathrm{O}$ critério para adoção do melhor modelo foi através da avaliação dos parâmetros: coeficiente de determinação ( $\mathrm{R}^{2}$ e $\mathrm{R}^{2}$ ajustado), erro padrão da estimativa $\left(\mathrm{S}_{\mathrm{yx}}\right)$, o valor de $\mathrm{F}$ calculado, o AIC, e o histograma de frequência dos erros. Para os mesmos modelos, utilizando a variável binária (dummy), os parâmetros de comparação foram o erro padrão da estimativa (Syx), o Akaike Information Criterion (AIC), este último como critério mais relevante na escolha do melhor modelo, por realizar análise mais justa entre modelos com mais ou menos variáveis e, penalizar aqueles com mais variáveis.

Tabela 1 - Nomenclaturas das variáveis de interesse em função das respectivas variáveis binárias.

\begin{tabular}{lcc}
\hline Variáveis de interesse & $\begin{array}{c}\text { Cód. da } \\
\text { variável }\end{array}$ & $\begin{array}{c}\text { Variável binária } \\
\text { (dummy) }\end{array}$ \\
\hline Peso da matéria seca da parte aérea (PMSPA) & $\mathrm{V} 1$ & D1 \\
Peso da matéria seca da raiz (PMSR) & V2 & D2 \\
Peso da matéria seca total (PMST) & V3 & D3 \\
Índice de qualidade de Dickson (IQD) & V4 & D4 \\
\hline
\end{tabular}

1)

2)

3)

4)

5)

6)

$$
\begin{gathered}
V_{k}=\beta_{0}+\beta_{1} D^{2} t x \\
V_{k}=\beta_{0}+\beta_{1} D t x+\beta_{2} D^{2} t x \\
V_{k}=\beta_{0}+\beta_{1} D t x+\beta_{2} H t x \\
V_{k}=\beta_{0}+\beta_{1} D t x+\beta_{2} H t x+\beta_{3} N F t x+\varepsilon \\
V_{k}=\beta_{0}+\beta_{1} D^{2} t x+\beta_{2} D^{2} H t x+\beta_{3} H t x+\varepsilon \\
V_{k}=\beta_{0}+D t x^{\beta_{1}}+H t x^{\beta_{2}}+\varepsilon
\end{gathered}
$$


em que: $\mathrm{V}_{\mathrm{k}}=$ Variáveis dependentes do modelo $(\mathrm{V} 1, \mathrm{~V} 2 \ldots \mathrm{Vn}) ; \beta_{0} ; \beta_{1} ; \ldots ; \beta \mathrm{n}=$ coeficientes estimados; $\mathrm{D}=$ diâmetro a altura do colo $(\mathrm{cm}) ; \mathrm{H}=$ altura $(\mathrm{cm}) ; \mathrm{NF}=$ número de folhas; $\mathrm{tx}=$ variável binária 0 e 1 (D1, D2... Dn).

Foi realizada também a correlação entre as variáveis de interesse utilizando o método de Pearson e assim gerada uma tabela matriz com os resultados. Todas as operações foram executadas no programa RStudio v. 1.1.423, por meio do pacote stats.

\section{RESULTADOS E DISCUSSÃO}

\section{Avaliação da altura e diâmetro a altura do colo}

Foi possível observar que as mudas produzidas em viveiro obtiveram valores médios em altura nas três avaliações de 21,90 cm, 18,97 cm e 23,25 cm; para diâmetro do colo 4,85, 4,65 e 5,38mm, respectivamente (Figura 1). Como as mudas foram selecionadas aleatoriamente em cada avaliação é comum que possa ocorrer decréscimo em uma ou mais medições devido as variações naturais no crescimento, e isso contribui na confiabilidade do parâmetro para expedição das mudas por considerar que o lote é heterogêneo.

De acordo com Gonçalves et al. (2000) [23], muda de boa qualidade deve apresentar altura variando de 20 a $35 \mathrm{~cm}$ e diâmetro do colo entre 5 e $10 \mathrm{~mm}$. Por outro lado, outros autores sugerem que mudas de espécies arbóreas estão aptas para o plantio no campo quando a altura da parte aérea estiver entre 15 e $30 \mathrm{~cm}$ [19]. Ainda assim, o Ministério de Agricultura, Pecuária e Abastecimento, por meio da implementação da Lei de Sementes e Mudas de Espécies Florestais (Lei 10.711, Decreto 5.153 de 2004) tem sugerido para várias espécies florestais nativas e exóticas padrão mínimo de $3 \mathrm{~mm}$ de diâmetro do colo e $20 \mathrm{~cm}$ de altura [24]. Entretanto, ainda é necessário que as mudas sejam avaliadas em campo para a determinação da qualidade de mudas sobre a manifestação dos parâmetros observados inicialmente em viveiro.
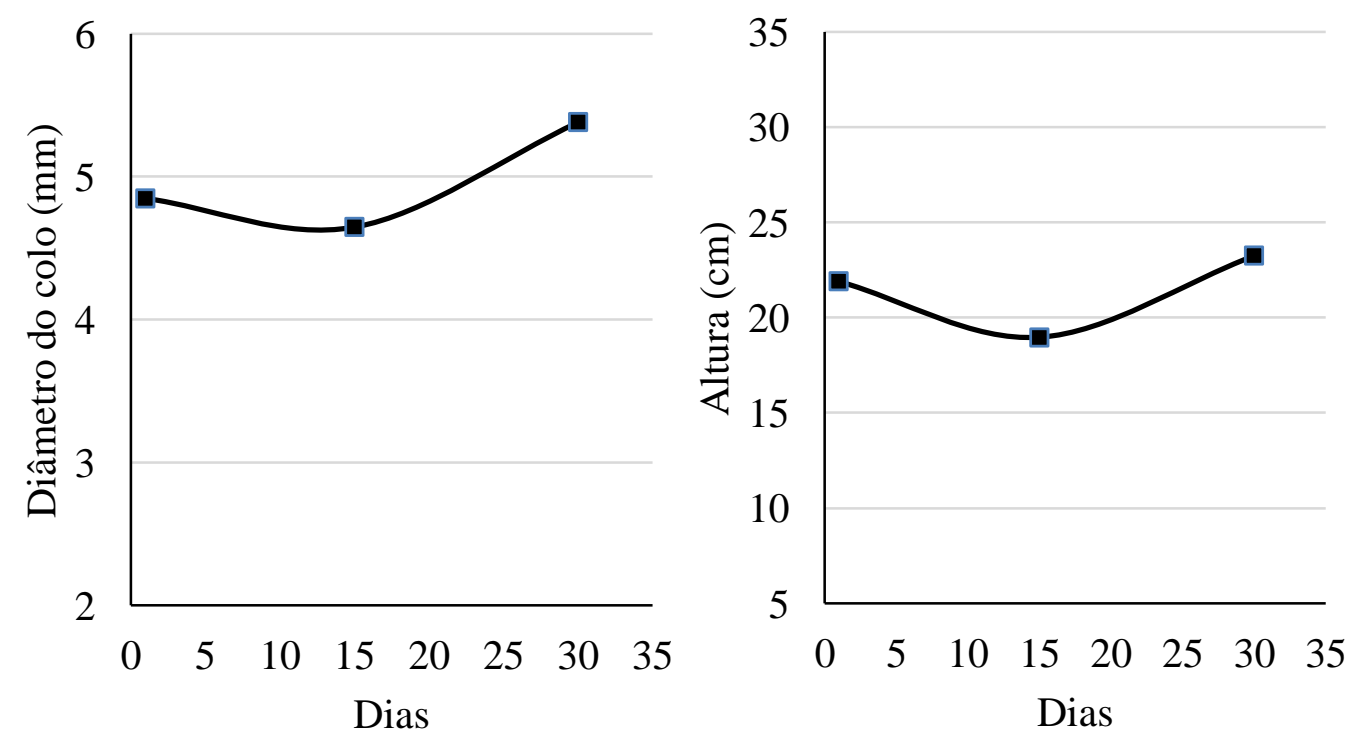

Figura 1 - Avaliações da altura da parte aérea $(H)$ e diâmetro do colo $(D A C)$ de mudas de Paubrasilia echinata (Lam.), com seus respectivos desvios, para avaliação da qualidade em viveiro.

Avaliando as informações apresentadas, tanto para a variável altura quanto para diâmetro do colo, as mudas alcançaram o padrão aceitável para plantio apenas na terceira avaliação após trinta dias desde a primeira análise. Nota-se também que houve acréscimo pouco significativo desde a primeira avaliação e a última, que pode ser explicado pelo crescimento lento da espécie por ser 
do grupo ecológico das climáxicas e semi-heliófita, segundo a classificação de Budowski (1965)[25].

\section{Avaliação do Peso de Matéria Seca da Parte Aérea (PMSPA), Peso de Massa Seca da Raiz (PMSR), Relação (PMSPA/PMSR) e Índice de Qualidade de Dickson (IQD).}

A relação PMSPA/PMSR foi satisfatória já que valor médio dessa relação atingiu a marca de 3,2, sendo o valor do índice que melhor representa essa relação igual a 2,0 [26] (Tabela 2). Segundo Caldeira et al. (2008)[13], para relação peso de massa seca de parte aérea e peso de massa seca de raízes nas mudas a proporção deve ser de 2:1, e a relação inversa deve ser de 1:2. Essa relação deve ser observada já que, a parte superior das mudas não deve ser expressivamente superior à área radicular, dificultando a absorção e transferência de água para a parte aérea principalmente em condições de campo, momento em que há maior exigência hídrica para pleno estabelecimento inicial do plantio.

Tabela 2 - Valores médios das variáveis de peso de massa seca da parte aérea (PMSPA), raiz (PMSR), total e Índice de Qualidade de Dickson (IQD) de mudas de Paubrasilia echinata (Lam.), São Cristóvão/SE.

\begin{tabular}{|c|c|c|c|c|c|}
\hline & \multicolumn{5}{|c|}{ Peso de massa seca (g) } \\
\hline & PMSPA & PMSR & PMSTotal & PMSPA/PMSR & IQD \\
\hline Média & 2,86 & 1,07 & 3,93 & 3,24 & 0,53 \\
\hline $\mathbf{S}$ & 1,49 & 0,78 & 2,22 & 1,22 & 0,29 \\
\hline$S^{2}$ & 2,21 & 0,62 & 4,92 & 1,49 & 0,09 \\
\hline CV\% & $51,98 \%$ & $73,41 \%$ & $56,44 \%$ & $37,62 \%$ & $54,88 \%$ \\
\hline IC & 0,92 & 0,49 & 1,38 & 0,76 & 0,18 \\
\hline
\end{tabular}

Com a espécie estudada, os valores mínimos e máximos desse índice foram de 0,22 e 1,03 respectivamente, e valor médio de 0,53 (Tabela 2). Hunt (1990) [27] sugere valor de IQD mínimo de 0,20 para mudas em tubetes, entretanto esse parâmetro não pode ser utilizado para mudas semeadas em sacos plásticos em detrimento do seu maior volume de substrato. Nesse sentido, o valor mínimo de IQD em torno de 0,35 pode ser um limite a ser considerado, de acordo com o intervalo de confiança encontrado, apesar da alta variação dessa variável nas mudas selecionadas.

Aguiar et al. (2011) [22], avaliando diferentes sombreamentos da mesma espécie obteve IQD médio de 3,46 e relação PMSPA/PMSR de 1,99 em mudas não sombreadas. Seu maior IQD pode ser explicado pelo maior período estudado (720 dias) além do maior número de mudas. No presente estudo as mudas foram acondicionadas em pleno sol, e o que se espera é comportamento diferenciado em espécies heliófitas como o pau-brasil. Isso justifica os valores da relação massa seca da parte aérea e raiz terem sido superiores, algo que pode ser associado ao comportamento fisiológico da espécie e sua estratégia de robustez na copa e raiz. Outro aspecto é que, as condições projetadas em viveiro florestal podem ter melhorado os parâmetros morfológicos das mudas.

\section{Equações para estimativa do Índice de Qualidade de Dickson (IQD), Peso da Massa Seca da Parte Aérea (PMSPA) e da raiz (PMSR).}

As equações ajustadas referentes ao IQD podem ser vistas na Tabela 3. Observa-se que dentre todos os modelos, as equações que obtiveram maior coeficiente de determinação ( $R^{2}$ aj.) foram as equações 3, 4 e 5. Isto demonstra que os modelos conseguem explicar a variável IQD e que a causa do maior valor de $\mathrm{R}^{2}$ na eq. 4 pode estar associada à inclusão da variável NF (número de folhas) no modelo. Dentro desse critério, a equação que obteve maior $\mathrm{R}^{2}$ aj., menor erro-padrão da estimativa (Syx) foi a equação 4, mas a inclusão de mais uma variável (NF) no modelo não conferiu melhores resultados em comparação a eq. 1.

O critério de informação de Akaike (AIC) evidencia o melhor desempenho para a eq. 1, apenas com o diâmetro do colo. Diferenças acima de 2 pontos ( $\Delta \geq 2$ ou critério canônico) conferem evidência suficiente de que os modelos não são iguais e, portanto, o que obtiver o menor valor 
será o modelo mais plausível para explicar o conjunto de dados fornecido [30]. Nesse sentido, a eq. 1 possuiu menor valor de AIC dentre todos os modelos avaliados o que atende ao critério canônico e, portanto, é diferente dos demais. Assim, a escolha de modelos com variáveis mais comuns e com relação biométrica conhecida é mais interessante nesse sentido, o que torna o modelo 1 mais conveniente do ponto de vista prático na coleta de informações das mudas.

Tabela 3. Coeficientes estimados para o Índice de Qualidade de Dickson (IQD) pelas variáveis:

Diâmetro do colo em cm (D); Altura em $\mathrm{cm}(H)$ e número de folhas $(N F)$ de mudas de Paubrasilia echinata (Lam.), São Cristóvão, SE.

\begin{tabular}{|c|c|c|c|c|c|c|c|c|c|}
\hline \multirow{2}{*}{ Eq. } & \multicolumn{4}{|c|}{ Coeficientes estimados } & \multirow{2}{*}{$\mathbf{R}^{2}$} & \multirow{2}{*}{$\mathbf{R}^{2} \mathbf{a j}$} & \multirow{2}{*}{ Syx } & \multirow{2}{*}{$\mathbf{F}$} & \multirow{2}{*}{ AIC } \\
\hline & $\boldsymbol{\beta}_{0}$ & $\beta_{1}$ & $\boldsymbol{\beta}_{2}$ & $\boldsymbol{\beta}_{3}$ & & & & & \\
\hline 1) & $-0,3957$ & $0,0389 *$ & & & 0,8033 & 0,7788 & 0,2978 & $32,68^{*}$ & 7,92 \\
\hline 2) & 0,2201 & $-0,2355$ & 0,0601 & & 0,8070 & 0,7519 & 0,3153 & $14,64 *$ & 13,73 \\
\hline 3) & $-1,0943$ & 0,24149 & 0,0384 & & 0,8486 & 0,8053 & 0,2793 & $19,61^{*}$ & 11,30 \\
\hline 4) & $-0,8709$ & 0,27547 & 0,0494 & $-0,1122$ & 0,8751 & 0,8127 & 0,2740 & $14,02^{*}$ & 17,38 \\
\hline 5) & $-2,3372$ & 0,4097 & $-0,0013$ & 0,1062 & 0,8634 & 0,7950 & 0,2866 & $12,64 *$ & 18,28 \\
\hline 6) & $-3,824 *$ & $0,5217 *$ & $0,2999 *$ & & 0,8460 & 0,8020 & 0,2817 & - & 11,47 \\
\hline
\end{tabular}

Os valores foram superiores aos encontrados por Binotto (2010) [28], ao utilizar o diâmetro do colo associado à altura ( $\mathrm{R}^{2}$ aj. 0,7693$)$ e que a variável $\mathrm{NF}$ combinada com as mesmas variáveis (D e H) apresentaram valores maiores $\left(\mathrm{R}^{2}\right.$ aj. 0,7860) para as espécies de Eucalyptus grandis Hill ex Maiden e Pinnus elliotti Engelm. A inclusão da variável NF para o presente estudo também apresentou maior rendimento nos valores de $R^{2}$ e $R^{2}$ aj na equação $4(0,8751$ e 0,8127 , respectivamente). Entretanto, mesmo que o NF tenha melhorado as estatísticas, a diferença não foi satisfatória e denota baixo poder da variável para explicar os eventos selecionados, vide alto valor do AIC em comparação a eq. 1 e os menores valores de correlação encontrados (Tabela 3 e 4).

Tabela 4 - Valores referente a correlação das variáveis pelo método de Pearson de mudas de Paubrasilia echinata (Lam.), São Cristóvão, SE.

\begin{tabular}{lccc}
\hline Variáveis & DAC & Alt & NF \\
\hline MSPA & 0,8880 & 0,9711 & 0,7502 \\
MSPR & 0,8171 & 0,8743 & 0,5721 \\
MST & 0,8816 & 0,9582 & 0,7088 \\
IQD & 0,8820 & 0,8739 & 0,5967 \\
\hline
\end{tabular}

A análise da distribuição de frequência dos erros percentuais não foi conclusiva no sentido de observar a distribuição dos resíduos (Figura 2). Não houve nítida diferença na distribuição em 5 dos 6 modelos, exceto na eq. 4. Em análise geral, os modelos possuíram leve assimetria à esquerda, ou seja, possui a capacidade de superestimar os valores de IQD. No entanto, observase que os erros ficaram concentrados entre -30 e $30 \%$, o que pode ser considerado satisfatório no quesito preditivo, sem perdas de informação. 
Modelo 1

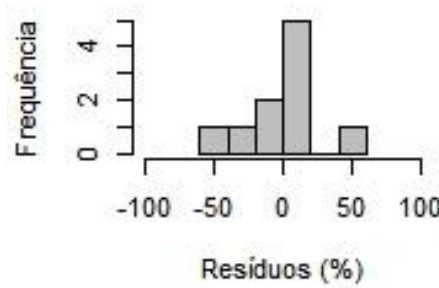

Modelo 4

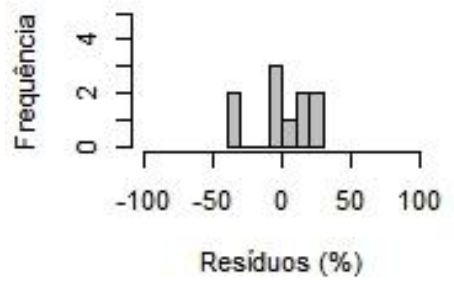

Modelo 2

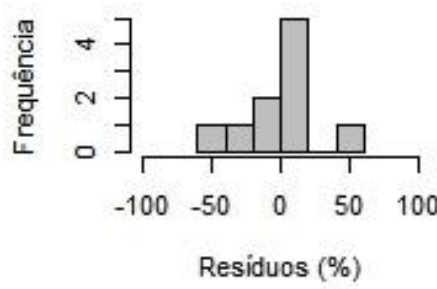

Modelo 5

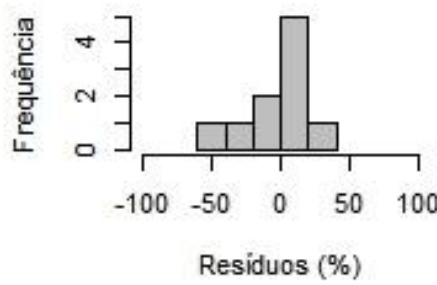

Modelo 3

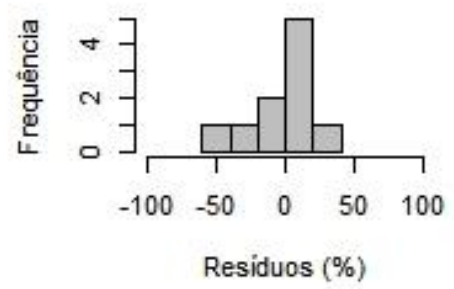

Modelo 6

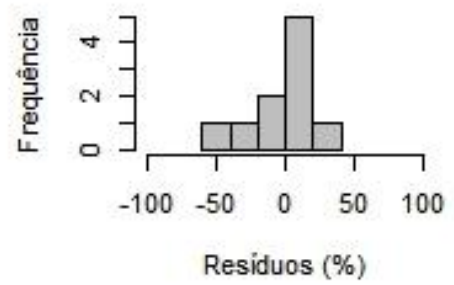

Figura 2 - Distribuição de frequência dos erros percentuais referente aos dois melhores modelos apresentados.

Em relação aos modelos com a inserção das variáveis binárias, a equação que obteve o menor erro foi a eq. 1 e, também, melhor valor de AIC (Tabela 5). Isto significa que as variáveis determinadas possuem relação quadrática com o diâmetro do colo das mudas e que a variável altura não foi significativa em 4 dos 6 modelos.

Tabela 5. Parâmetros dos modelos testados com a inclusão da variável fictícia, São Cristóvão, SE, 2013.

\begin{tabular}{lcc}
\hline Eq. & Syx & AIC \\
\hline 1$)$ & 1,1330 & 186,55 \\
$2)$ & 0,9675 & 252,60 \\
$3)$ & 0,7125 & 228,13 \\
$4)$ & 0,7409 & 341,09 \\
$5)$ & 0,7498 & 344,41 \\
$6)$ & 0,7510 & 232,34 \\
\hline
\end{tabular}

\section{CONCLUSÃO}

Com base na análise dos parâmetros morfológicos altura, diâmetro do colo e número de folhas, as mudas de pau-brasil estão aptas ao plantio após 215 dias, de acordo com o padrão estabelecido (DAC $\geq 5 \mathrm{~mm}$ e $\mathrm{H} \geq 20 \mathrm{~cm}$ ).

$\mathrm{Na}$ avaliação da massa seca, a relação PMSPA/PMSR foi satisfatória atingindo valor acima de 2,0, como recomendado na literatura. Entretanto, para o Índice de Qualidade de Dickson (IQD), as mudas apresentaram valor não aceitável e, portanto, necessita de maiores estudos com a espécie avaliando seu desempenho em campo.

O diâmetro na altura do colo mostrou forte correlação para explicar o IQD, e apresenta relação quadrática com esta variável.

As equações apresentaram ajustes aceitáveis, considerando as limitações amostrais no número de mudas abatidas, sendo, portanto, uma ferramenta a ser considerada na tomada de decisões 
quando não há a possibilidade de determinação da massa seca por métodos destrutivos. No entanto, estudos futuros podem incluir outras variáveis a exemplo da relação $\mathrm{H} / \mathrm{D}$, dias após a emergência (DAE), parâmetros de qualidade de sementes e taxa fotossintética foliar, auxiliando e gerando informações importantes a serem consideradas em programas que visam a recuperação de áreas degradadas, principalmente utilizando-se espécies nativas ameaçadas de extinção.

\section{REFERÊNCIAS BIBLIOGRÁFICAS}

1. Borges LA, Sobrinho MS, Lopes AV. Phenology, pollination, and breeding system of the threatened tree Caesalpinia echinata Lam. (Fabaceae), and a review of studies on the reproductive biology in the genus. Flora, 2008;204:111-130, doi:org/10.1016/j.flora.2008.01.003

2. Lamarca EV, Leduc SNM, Barbedo CJ. Viabilidade e vigor de sementes de Caesalpinia echinata Lam. (pau-brasil - Leguminosae) pelo teste de tetrazólio. Rev Bras Bot. 2009 Out/Dez;32(4):793-803.

3. Gagnon E, Bruneau A, Hughes CE, Queiroz L, Lewis GP. A new generic system for the pantropical Caesalpinia group (Leguminosae). PhytoKeys. 2016;71:1-160, doi:10.3897/phytokeys.71.9203.

4. Elias GA, Padilha, PT, Santo R. Biodiversidade em Santa Catarina: Parque Estadual da Serra Furada. UNESC. Criciúma:SC; 2016. 188 p.

5. CNCFlora, 2012. Caesalpinia echinata in Lista Vermelha da flora brasileira versão 2012.2 Centro Nacional de Conservação da Flora. Acesso em 23 de abril 2017. Disponível em http://cncflora.jbrj.gov.br/portal/pt-br/profile/Caesalpinia echinata.

6. Lima HC. Taxonomia e distribuição geográfica atual de Caesalpinia echinata Lam. In: Simpósio "Paubrasil: Ciência e Arte". FAPESP. 2003. São Paulo CD ROM.

7. Valeri SV, Sá AFL, Martins ABG, Barbosa JC. Enraizamento de estacas de Caesalpinia echinata Lam. em hidroponia. Ci Fl. 2012 Abr/Jun;22(2):241-250, doi:10.5902/198050985731.

8. Garcia IS, Souza A, Barbedo CJ, Dietrich SMC, Figueiredo-Ribeiro RCL. Changes in soluble carbohydrates during storage of Caesalpinia echinata LAM. (Brazilwood) seeds, an endangered leguminous tree from the Brazilian Atlantic Forest. Brazilian J Biol. 2006 Maio;66(2B):739-745.

9. Endres L, Marroquim PMG, Santos CMD, Souza NNFD. Rooting cuttings of Pau-Brasil (Caesalpinia echinata Lam.) treated with indole butyric acid and naphthalene acetic acid. Ci Rural. 2007 Mai/Jun;37(3):886-889.

10. Tofanelli MBD, Chalfun NNJ, Hoffmann A, Chalfun Júnior A. Efeito do ácido indolbutírico no enraizamento de estacas de ramos semilenhosos de pessegueiro. Pesq Agrop Bras. 2002 Jul;37(7): 939944, doi:10.1590/S0100-204X2002000700007

11. Fernandes LA, Furtiniti Neto A F, Vale FR. Crescimento inicial, níveis críticos de fósforo e frações fosfatadas em espécies florestais. Pesq Agrop Bras. 2000 Jun;35(6):1191-1198.

12. Ajalla ACA, Vieira MC, Volpe E, Zárate NAH. Crescimento de mudas de Campomanesia adamantium (Cambess.) O. Berg (guavira), submetidas a três níveis de sombreamento e substratos. Rev Bras Frutic. 2014 Jun;36(2):449-458, doi:10.1590/0100-2945-196/13

13. Caldeira MVW, Rosa GN, Fenilli TAB, Harbs, RMP. Composto orgânico na produção de mudas de aroeira-vermelha. Scientia Agraria. 2008;9(1):27-33, doi:10.5380/rsa.v9i1.9898

14. Gomes JM. Parâmetros morfológicos na avaliação da qualidade de mudas de Eucalyptus grandis, produzidas em diferentes tamanhos de tubetes e de dosagens de NP-K. [Tese]. Viçosa (MG): Universidade Federal de Viçosa; 2001. 126p.

15. Eloy E, Caron BO, Schmidt D, Behling A, Schwers L, Elli EF. Avaliação da qualidade de mudas de Eucalyptus grandis utilizando parâmetros morfológicos. Floresta. 2013 Jul/Set;43(3):373-384.

16. Chaves AS, Paiva HN. Influência de diferentes períodos de sombreamento sobre a qualidade de mudas de fedegoso (Senna macranthera (Collad.) Irwin et Barn.). Scientia Forestalis. 2004 Jun;(65):22-29.

17. Novaes AB, Silva HF, Sousa GTO, Azevedo, GB. Qualidade de mudas de Nim Indiano produzidas em diferentes recipientes e seu desempenho no campo. Floresta. 2014 Jan/Mar;44(1):101-110, doi:10.5380/rf.v44il.30207

18. Luca EF, Rebecchi RJ, Schorn LA. Crescimento e qualidade de mudas de cedro (Cedrela fissilis Vellozo) em viveiro, mediante diferentes técnicas de produção. Rev Inst Fl. 2010 Dez;22(2):189-199.

19. Gomes JM, Paiva HN. Viveiros florestais: [propagação sexuada]. 3.ed. Viçosa - Mg: UFV, Il. (Cadernos Didáticos; 72); 2004. 116p.

20. Laar AV, Akça, A. Forest Mensuration: Managing Forest Ecosystems. 2nd. Ed. Springer: vol. 13; Von Gadow K, Pukkala T, Tomé M. (Ed.). 2007. 383p.

21. West PW. Tree and Forest measuration. 2nd edition - Springer-Verlag Berlin Heidelberg, 2009. 190p, doi:10.1007/978-3-540-95966-3_14 
22. Aguiar FFA, Kanashiro S, Tavares AR, Nascimento TDR, Rocco FM. Crescimento de mudas de paubrasil (Caesalpinia echinata Lam.), submetidas a cinco níveis de sombreamento. Ceres. 2011 Nov/Dez;58(6):729-734, doi:10.1590/S0034-737X2011000600008

23. Gonçalves JLM, Santarelli EG, Moraes Neto SP, Manara, M.P. Produção de mudas de espécies nativas: substrato, nutrição, sombreamento e fertilização. In: Gonçalves JLM, Benedetti V. (Eds.) Nutrição e fertilização florestal. Piracicaba: ESALQ/USP; 2000. p. 309-350.

24. Pacheco BM. Caracterização do ciclo de produção de mudas de espécies florestais nativas do estado de Sergipe. [Monografia]. São Cristóvão (SE) Universidade Federal de Sergipe; 2010. 32p.

25. Budowski A. Distribution of tropical rain forest species in the light of successional progress. Rev. Turrialba. 1965;15(1):40-42.

26. Brissette JC. Summary of discussions about seedling quality. In: Southern Nursery Conferences, Alexandria. Proceedings. New Orleans: USDA. Forest Service. Southern Forest Experiment Station; 1984. p. 127-128.

27. Hunt GA. Effect of styroblock design and cooper treatment on morphology of conifer seedlings. In: Target Seedlings Symposium, Meeting Of The Western Forest Nursery Associations, Roseburg, 1990. Proceedings. Fort Collins: United States Department of Agriculture, Forest Service; 1990. p.218-222.

28. Binotto AF, Lúcio DCA, Lopes SJ. Between growth variables and the Dickson quality índex in Forest seedling. Cerne. 2010 Out/Dez;16(4):457-464.

29. Melo AS, Neto AOA, Neto JD, Brito MEB, Viegas PRA, Magalhães LTS, Fernandes PD. Desenvolvimento vegetativo, rendimento da fruta e otimização do abacaxizeiro cv. Pérola em diferentes níveis de irrigação. Ci Rural. 2006;36(1):93-98.

30. Burnham KP, Anderson DR. Model selection and multimodel inference: A practical informationtheoretic approach. Springer Science \& Business Media: Colorado; 2002. 488p. doi:10.1007/B97636

31. Faria JMR. Propagação de espécies florestais para recomposição de matas ciliares. In: Simpósio Mata Ciliar: ciência e tecnologia, 1999, Belo Horizonte. Anais..., Belo Horizonte; 1999. p. 69-79. 